REVISION DE TEMA

\title{
Asociación entre hiperhomocisteinemia y preeclampsia
}

\author{
Jorge Mario Gómez Jiménez*, Jhon Jairo Zuleta Tobón*, Germán Campuzano Mayo**, Carlos Mario \\ Córdoba Gómez*** \\ Recibido: Agosto 17/2001 - Revisado: Noviembre 4/2001 - Aceptado: Febrero 11/2001
}

\begin{abstract}
RESUMEN
OBJETIVO: Explorar la asociación entre el nivel sérico posprandial de homocisteína y la preeclampsia y describir las complicaciones en gestaciones menores de 35 semanas.

METODOLOGÍA: Estudio descriptivo de corte transversal en 109 gestantes: grupo I, 55 preeclámpticas (90,9\% clasificadas como severas) y grupo II, 54 sin preeclampsia con menos de 35 semanas de edad gestacional, de los hospitales Universitario San Vicente de Paúl y General de Medellín, entre $1^{\circ}$ de septiembre de 2000.
\end{abstract}

RESULTADOS: Cuando se compararon las características generales de las pacientes no se encontró diferencia. No se demostró correlación entre los niveles de homocisteína y la edad de la paciente, la edad gestacional, ni con el número de partos previos (rho de Spearmman-0,057, 0,074 y - 0,17 respectivamente).

Hubo diferencia entre las medianas de las concentraciones de homocisteinemia: 11,8 micromol/L y 8,8 micromol/L para G I y G II respectivamente ( $p<$ de 0,01 ). Las pacientes con homocisteinemia en el último cuartil (concentración mayor de 11,2 micromol/L por inmunoensayo de fluorescencia polarizada) presentan un riesgo de tener preeclampsia 9,67 veces mayor que las del primer cuartil (concentración menor de 7,1) $\left(<\mathrm{IC}_{95 \%}\right.$ entre 4,48 y 142,66).

El percentil 95 para la homocisteinemia en embarazadas normotensas fue de 13,025 micromol/L. Se estableció este valor como el punto de corte para diagnosticar hiperhomocisteinemia en la gestante. Veinticinco (45.5\%) de las gestantes del grupo I y 2 (3.7\%) del grupo II fueron clasificadas con hiperhomocisteinemia, razón de disparidad (RD) 21,67 (IC95\% entre 4,48 y 142,66).

Catorce pacientes del grupo I tuvieron hijos con RCIU. Trece resultaron de madres con hiperhomocisteinemia frente a 12 de las 30 restantes cuyos hijos nacieron con peso adecuado para la edad gestacional (PAEG) $(\mathrm{p}=0,37)$. Seis neonatos, hijos de 26 mujeres no hiperhomocisteinémicas del grupo II que terminaron su gestación en los hospitales donde se realizó el estudio, presentaron bajo peso para la edad gestacional.

CONCLUSIÓN: Se definió hiperhocisteinemia en la gestante si el nivel sérico posprandial de homocisteína resultó ser igual o mayor de 13,025 micromol/L (por encima del percentil 95 para el grupo II) y se demostró asociación (no causal), con preeclampsia.

PALABRAS CLAVE: preeclampsia, homocisteína, hiperhomocisteinemia.

\footnotetext{
* Ginecoobstetra, Docente Universidad de Antioquia.

** Hematólogo Clínico, Laboratorio Clínico Hematológico.
} 


\section{SUMMARY}

OBJECTIVE: To explore the association between posprandial elevated serum levels of homocystein and preeclampsia and related complications before 35 weeks of gestational age.

METHODOLOGY: Cross sectional study on 109 pregnant patients before 35 weeks of gestational age (group I, $\mathrm{n}=55$ pre-eclamptic patients (90,9\% severe cases), and group II, $\mathrm{n}=$ 54 non pre-eclamptic patients) of the hospitals Universitario San Vicente de Paúl and General de Medellín, from September $1^{\text {st }}, 1999$ to September $1^{\text {st }}, 2000$.

RESULTS: There were no differences between both groups when analyzing general characteristics. There was no correlation between homocystein, measured by fluorescent polarized immunoassay (FPIA) with pregnant patient age, gestational age no parity (Spearman's rho $-0,057-0,074-0,17$ respectively).

There was a significant difference between medians of the homocystein concentration in compared groups: in G I it was 11,8 and 8,8 micromol/L in GII $(p<0,01)$.

Patients with homocystein level in the last quartile ( $>11.2$ micromol/L) have a risk 9,67 times higher when compared with patients in the first quartile (Homocystein $<7,1$ ) to present preeclampsia $(p=0,001)$. Percentile 95 for norm-tense pregnant patients was 13,025 micromol/L. This level was established like an end point for hyperhomocysteinemia definition in pregnant women; 25 (45,5\%) in G I and 2 (3,37\%) in G II of the patients were positive for hyperhomocysteinemia, odds ratio (OR) 21,67 (confidence interval 95\% 4,48 - 142,66, $p<$ 0,001).

Fourteen group I patients had complications attributable to preeclampsia, 6 were positive women for hyperhomocysteinemia compared to 19 positive patients for hyper homocysteinemia of 41 without complications in the same group $(p=0,397)$.

Thirteen of 25 intrauterine growth restriction (IUGR) events in G I compared to 12 patients of 30 without IUGR, were positive patients for hyperhomocysteinemia $(\mathrm{p}=0,37)$. Six newborns of 26 women in group II that ended gestation in the source hospitals, presented intrauterine grow restriction but their mothers were negative for hyperhomocysteinemia.

CONCLUSION: hyperhomocysteinemia was defined in pregnancy above p95, HC $\geq$ $13,025 \mathrm{micromol} / \mathrm{L}$ and an association, though not causal, between hyperhoocysteinemia and preeclampsia was found.

KEY WORDS: preeclampsia, homocystein, hyperhomocysteinemia.

\section{INTRODUCCIÓN}

La preeclampsia es una de las tres principales causas de mortalidad materna en el mundo y es la enfermedad obstétrica más importante en muchos países ${ }^{1}$. En Colombia, según estadísticas del Ministerio de Salud, ocupa el primer puesto en mortalidad materna con 42 por 100.000 nacidos vivos ${ }^{2}$. Adicionalmente, produce complicaciones maternas como falla renal, coagulopatía, convulsiones y síndrome Hellp y fetales como retardo del crecimiento intrauterino (RCIU), sufrimiento fetal agudo (SFA), prematurez, y muerte fetal.

La preeclampsia es llamada la enfermedad de las teorías (genética, inmunológica, endotelial, etc) porque todavía se desconoce su causa ${ }^{3}$. Se puede afirmar que su etiología es multifactorial y, por lo que se sabe hoy, la inmunológica es una de las que más se acerca a integrar el origen de esta entidad ${ }^{4}$. Esta teoría se basa 
en las características individuales de su presentación de la enfermedad tales como su ocurrencia en primigestantes, en multíparas con hijo de compañero sexual diferente a los anteriores, su asociación con otras entidades clínicas donde el tamaño de la placenta es mayor como en diabetes gestacional, embarazo gemelar, mola hidatidiforme, degeneración hidrópica placentaria y triploidía. Se presenta por mecanismos patogénicos comunes tales como el vasoespasmo, la activación de neutrófilos y el daño endotelial ${ }^{5}$.

Investigaciones recientes sugieren asociación entre preeclampsia y otras trombofilias diferentes al síndrome antifosfolípido. Sorprenden por su frecuencia: deficiencia de proteina $S$, resistencia a la proteína C activada e hiperhomocisteinemia ${ }^{6-8}$.

Los objetivos del presente estudio fueron buscar la asociación entre la presencia de preeclampsia y los niveles de homocisteína en sangre y relacionarlos con las complicaciones maternas y fetales con el propósito de buscar posibles intervenciones nutricionales o farmacológicas que mejoren el pronóstico de estas pacientes.

\section{MATERIALES Y MÉTODOS}

Tipo de estudio: Descriptivo de corte transversal (cross - sectional).

Población de referencia: Gestantes que consultaron en los servicios de urgencias y de alto riesgo obstétrico de los hospitales San Vicente de Paúl y General de Medellín, con preeclampsia moderada o severa (grupo I) o con gestaciones no complicadas por trastornos hipertensivos (grupo II) antes de las 35 semanas de gestación.

Muestra: A conveniencia. Se emplearon los sueros de 109 pacientes y los datos pertinentes de sus historias clínicas que se habían seleccionado previamente para un estudio de asociación entre anticuerpos antifosfolípidos y preeclampsia (en prensa).

Muestreo: grupo I, 55 gestantes con preeclampsia (90,0\% preeclampsia severa) y el grupo II, 54 gestantes sin preeclampsia que tenían amenazada de parto prematuro (APP) (66\%) y ruptura prematura de membranas ovulares (RPMO) (44\%).
Criterios de exclusión: gestantes con diagnóstico de hipertensión arterial crónica, colagenopatía, nefropatía crónica, embarazo múltiple, embarazo molar, tiroidopatía, epilepsia, antecedente personal de aborto recurrente, feto malformado o muerto in útero en la gestación índice. También se excluyeron las mujeres que recibían cualquiera de estos medicamentos: clorpromazina, ácido valproico, hidralazina, amoxicilina, propanolol, estreptomicina, procainamida, interferón, quinidina, sulfadoxina, dilantín, primetamina.

Criterios de inclusión: se incluyeron gestantes en el grupo I, si la presión anterial se encontró mayor o igual a 140/90 mmHg en dos oportunidades, separadas por seis horas, con proteinuria mayor o igual a 300 $\mathrm{mg}$ en la orina de 24 horas. Se clasificó preeclampsia severa si se presentaron signos y síntomas premonitorios de convulsión, deterioro renal definido por oliguria (diuresis $\leq 720 \mathrm{cc} / 24$ horas), HELLP y RCIU. Sólo se incluyeron las pacientes que cumplieron estos criterios y que se encontraron con feto vivo in útero sin malformaciones. Para el grupo II se incluyeron gestantes con feto vivo no malformado, sin preeclampsia.

Los criterios para el diagnóstico de HELLP fueron: crenocitos en extendido de sangre periférica o deshidrogenasa láctica mayor o igual a 1000 U/lt, aspartaloaminotransferasa $\quad 0$ alaninoaminotransferasa mayor o igual a 70 $\mathrm{U} / \mathrm{L}$ y recuento de plaquetas menor o igual a $100.000 / \mathrm{mL}$.

El RCIU fue definido como peso fetal estimado por perímetro abdominal en la ultrasonografía fetal, por debajo del percentil 10 de Hadlock para la edad gestacional definida por anemone confiable y/o ultrasonografía en el primer trimestre de la gestación. Se aplicó el mismo criterio para definir RCIU después del parto.

La edad gestacional fue definida por la anemone confiable en semanas o en su defecto por la edad gestacional encontrada por ultrasonografía fetal del primer trimestre de la gestación.

Especificaciones técnicas de la prueba de laboratorio: a los sueros que habían sido congelados después de la realización de una investigación previa se les realizó medición de los niveles de 
homocisteína mediante inmunoensayo de fluorescencia polarizada desarrollado por Axis Biochemical ASA de Oslo, Noruega y Bio-Rad Hércules, California, comercializado y distribuido por Abbott Laboratories empleando un analizador Imx - System. Las muestras se recolectaron dos horas después de un desayuno bajo en grasa, sangrando de vena periférica 10 cc en tubo seco. Después de separar el coágulo se extrajo el suero por centrifugación de gravedad y se congeló a $-20^{\circ} \mathrm{C}$. Estuvieron congelados por diez meses. El laboratorio realizó confirmación de todos los valores anormales. Las pruebas fueron realizadas en el Laboratorio Clínico Hematológico Ltda., Medellín - Colombia.

Consideraciones éticas: Este proyecto tuvo la aprobación del comité de Ética de los hospitales participantes. Todas las pacientes firmaron consentimiento para su participación, además, donaron sus sueros para futuras investigaciones.

Análisis estadístico: se utilizaron los programas SPSS versión 8,0 y Epiinfo 6. Se evaluó la normalidad de la distribución de las variables continuas mediante la prueba de Kolmogorov - Smirnov; como se encontró que no seguían esta distribución se realizó comparación de los grupos con la prueba U de Mann Witney. Para las variables cualitativas se utilizó la prueba de

Tabla 1

Características generales de la población

Asociación entre hiperhomocisteinemia y preeclampsia. Medellin 2000 chi cuadrado y para los datos en escala ordinal se empleó la prueba chi cuadrado de tendencia.

\section{RESULTADOS}

No hubo diferencias entre los grupos en cuanto a las características generales (tabla 1). No existe correlación entre los niveles de homocisteína y la edad de la paciente (rho de Spearman -0,057), la edad gestacional (rho - 0,074) ni con el número de partos previos (rho - 0,17).

La distribución de las concentraciones de homocisteína en el grupo I no es gaussiana ( $p \quad 0.003$ en la prueba de Kolmogorov-Smirnov). Varía enre $3.8 \mathrm{y}$ $49.7 \mathrm{micromol} / \mathrm{L}$ con media de 13,4 y mediana de 11.8. En el grupo II, la concentración de homocisteína sigue una distribución gausiana o normal ( $p=0.2$ en la misma prueba) con una media de $9.1 \mathrm{y}$ desviación estándar (ds) de 2.9, valor mínimo 3.7 y máximo 21.77.

Hubo diferencia estadísticamente significativa enre las medianas de homocisteína de los grupos comparados. La mediana de la homocisteína para el grupo I fue de 11,8 micromol/L y de 8,8 para el grupo II ( $p$ $<$ de 0,01) (gráfico 1).

\begin{tabular}{|lccc|}
\hline Variable & Grupo I n=55 & Grupo $\mathbf{~} \mathbf{~ n = 5 4}$ & \multicolumn{1}{c|}{$\boldsymbol{P}$} \\
\hline Edad $^{(1)}$ (años) & $26 \pm 7$ & $25 \pm 7$ & $0,48^{(2)}$ \\
Edad Gestacional (semanas) $^{(1)}$ & $30,4 \pm 2,6$ & $31 \pm 2,7$ & $0,29^{(2)}$ \\
${\text { Edad Gestacional }<28 \text { semanas }^{(4)}}^{(17(31 \%)}$ & $9(16,7 \%)$ & $0.081^{(3)}$ \\
Gravidez 1 & $21(38,2)$ & $16(29,6)$ & \\
2 & $8(14,6)$ & $18(33,3)$ & $0,082^{(3)}$ \\
3 & $15(27,2)$ & $8(14,9)$ & \\
4 & $11(20,0)$ & $12(22,2)$ & \\
\hline
\end{tabular}

${ }^{1}$ Promedio y desviación estándar

2 t student

3 c 2

4 número (\%) 
Tabla 2

Asociación entre niveles séricos de homocisteina* por cuartiles y preeclampsia Asociación entre hiperhomocisteinemia y preeclampsia. Medellin 2000

\begin{tabular}{|cccccc|}
\hline CUARTI & RANGO & GRUPO I N = 55 & GRUPO $\mathbf{~} \mathbf{N}=\mathbf{5 4}$ & RD+ & IC $_{\mathbf{9 5} \%}$ \\
\hline 1 & $<7,1$ & 3 & 13 & 1,00 & (grupo de \\
& $7,1-8,6$ & & & 4,02 & referencia) \\
2 & $9,6-11,2$ & 13 & 14 & 3,10 & $0,79-22,97$ \\
3 & & 10 & 14 & 9,67 & $0,58-18,29$ \\
4 & $>11,2$ & 29 & 13 & & $2,04-52,17$ \\
\hline
\end{tabular}

${ }^{+} \mathrm{RD}=$ Razón de disparidad

* Homocisteina sérica posprandial en umol/L

Se tomó como grupo de referencia para calcular las razones de disparidad (RD) el percentil 25 del grupo de pacientes normotensas (concentración menor de 7,1 micromol/L). Las pacientes con un nivel de homocisteína en el percentil 75 (concentración mayor de 11,2 mol/L) tienen un riesgo de 9,67 veces más de tener preeclampsia (IC 95\% $2.04 \quad-52.17$ ) comparadas con las del percentil 25 ( $p=$ 0.001) (tabla 2).

El percentil 95 para las embarazadas normotensas fue de 13,025 micromol/L; si se establece este valor como el punto de corte para diagnosticar Hiperhomocisteinemia en el embarazo, el $45,5 \%$ del grupo I serían catalogadas como tales, RD 21,67 (IC 95\% 4,48 - 142,66) ( $p$ menor de 0,001) (Gráfico 2).

Catorce pacientes del grupo I tuvieron complicaciones atribuibles a preeclampsia, 6 presentaron hiperhomocisteinemia frente a 19 de 41 pacientes que no se complicaron en este grupo. No se demostró evidencia de asociación entre niveles elevados de hiperhomocisteinemia complicaciones atribuibles a preeclampsia $(\mathrm{p}=0,397)$ (Tabla 3).

Se presentaron 25 casos de RCIU en el grupo I, 13 tenían niveles elevados de homocisteína y 12 niveles normales. No se demostró evidencia e asociación entre niveles elevados de homocisteinemia $y$ RCIU (p = 0,37). Seis neonatos, hijos de 26 mujeres del grupo II que terminaron su gestación en los hospitales donde se realizó el estudio, presentaron RCIU; sus madres fueron negativas para el marcador estudiado.
Tabla 3

Complicaciones en preeclámpticas con hiperhomocisteinemia Asociación entre hiperhomocisteinemia* y preeclampsia. Medellin, 2000

\begin{tabular}{|llc|}
\hline CASO & COMPLICACION & HC \\
\hline 1 & Sindrome HELLP* - Abrupcio Placenta - & 7,7 \\
& Edema pulmonar & \\
2 & Eclampsia & 9,2 \\
3 & Abrupcio placenta & 9,8 \\
4 & Sindrome HELLP & 7,8 \\
5 & Sindrome Nefrótico & 3,8 \\
6 & Sindrome HELLP* - Edema pulmonar & 12,5 \\
7 & Abrupcio placenta & 8,3 \\
8 & Muerte Fetal* & 26,4 \\
9 & Abrupcio placenta * & 14,9 \\
10 & Insuficiencia Renal Aguda & 6,1 \\
11 & Muerte Fetal* & 15,6 \\
12 & Sufrimiento fetal crónico & 49,7 \\
13 & Sufrimiento fetal agudo & 20,0 \\
14 & Sufrimiento fetal crónico & 9,5 \\
\hline
\end{tabular}

- Complicación y Homocisteina sérica posprandial $\geq 13,025 \mathrm{mmol} / \mathrm{L}$ * HELLP = Hemólisis - Enzimas hepáticas elevadas - Plaquetas disminuidas

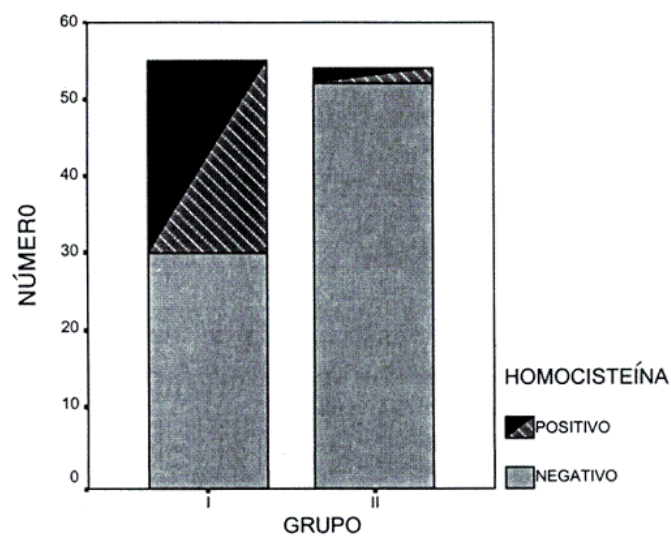

* Punto de corte en $13,025 \mu \mathrm{mol} / \mathrm{L}$

Figura 001 i02

Hipermocisteinemia en pacientes preeclámpticas y no preeclámpticas. Asociación entre hiperhomocisteinemia y preeclampsia. Medellín. 2000 
Sólo una de las gestantes del grupo II en quien después se demostró estenosis mitral, se complicó con tromboembolismo pulmonar demostrado por gamagrafía; sobrevivió con anticoagulación.

\section{DISCUSIÓN}

Por el tipo de trabajo fue posible definir asociación entre hiperhomocisteinemia y preeclampsia severa pero no se puede inferir causalidad porque fueron medidos en el mismo instante. No es posible saber si los niveles elevados de homocisteína son un factor causal o solo una consecuencia de las múltiples que tiene la preeclampsia.

Pudiera proponerse que los niveles elevados de homocisteinemia son explicados por la hemoconcentración propia de la preeclampsia pero otros estudios previos ya han descartado esta posibilidad. Igualmente, las variaciones no pueden ser explicadas por la edad materna, la edad gestacional ni la paridad porque no se encontró correlación entre ellos.

El presente estudio hace parte de la continuación de otro previamente realizado para evaluar la asociación entre anticuerpos antifosfolípidos y preeclampsia y aunque el poder para detectar diferencias en el presente era solamente del $60 \%$, esta situación no afectó los resultados porque incluso con este tamaño muestral y este poder, las diferencias se hicieron evidentes.

Hubo pérdida de información del peso fetal del grupo II, que por ser partos de bajo riesgo, no son atendidos en las instituciones base de la investigación (son de tercer nivel). A pesar de los esfuerzos no fue posible recuperar esa información, por lo tanto, las conclusiones en relación con RCIU y su asociación con la hiperhomocisteinemia obtenidas en el presente trabajo no son válidas; es probable que si se tuviera la información de la totalidad de los recién nacidos se encontrarían diferencias entre los grupos.

Se presenta hiperhomocisteinemia en mujeres portadoras de una mutación en el gen que codifica la enzima Cistatión $\beta$ sintetasa, quienes tempranamente sufren retardo mental, luxación de cristalino, osteoporosis y escoliosis, trombosis $\mathrm{y}$ arterioesclerosis. Presentan niveles plasmáticos de homocisteína mayores de $100 \mathrm{micromol} / \mathrm{L}$ y tasa de mortalidad del
$50 \%$ antes de los 30 años $^{9-11}$. También se presenta por déficit nutricional 0 metabólico de las vitaminas $\mathrm{B}_{6} \mathrm{y}_{12}$, ácido fólico y de la enzima metilentetrahidrofolato reductasa.

Evidencias recientes definen la hiperhomocisteinemia como un factor de riesgo vascular independiente. Se ha asociado a trombosis venosa profunda, arterioesclerosis, enfermedad coronaria y accidente cerebrovascular. También se ha descrito correlación con aborto recurrente, muerte fetal, abrupcio de placenta y preeclampsia $^{11}$.

Según Pampus y colaboradores ${ }^{12}, 11$ de 58 preeclámpticas severas (19\%) antes de las 28 semanas de gestación, presentaron niveles altos de homocisteina frente a 3 de 67 controles $(4,5 \%)(p<0.05)$. Cuando la preeeclampsia se presentó después de las 28 semanas, 35 de 289 (10.4\%) presentaron niveles altos.

Rajkovic y colaboradores ${ }^{13}$, compararon los niveles de homocisteína plasmática entre 20 primigrávidas preeclámpticas y 20 normales al momento del parto, demostrando niveles más altos en las primeras, diferencia con significación estadística. Los mismos autores en otra investigación con gestantes africanas eclámpticas o preeclámpticas demostraron niveles elevados de homocisteína frente a testigos normotensas con razones de disparidad, (RD), de 6,03, (IC (I5\% $_{1,48}$ 24,52) y 4,57, $\quad\left(\mathrm{IC}_{95 \%} \quad 2,11 \quad-9,88\right)$ respectivamente $^{14}$.

Otras investigaciones asocian preeclampsia con resistencia a la insulina, niveles altos de homocisteinemia y con marcadores de daño endotelial. Igualmente con disbalance metabólico de compuestos que contienen átomos de azufre (glutatión, homocisteina y cistina) ${ }^{15-17}$.

Ray y Laskin $^{18}$ encontraron en una revisión de cinco estudios que la hiperhomocisteinemia se asocia con riesgo de desarrollar preeclampsia con RD de 20,9 ( IC $_{95 \%}$ 3,6 -121,6).

Wang $^{19}$ encontró concentraciones significativamente mayores de homocisteinemia en gestantes complicadas con preeclampsia 0 enfermedades vasculares placentarias cuando fueron comparadas con controles normotensas, 
sugiriendo a la hiperhomocisteinemia como marcador de riesgo para preeclampsia ${ }^{19}$.

Sorensen $\mathrm{y} \operatorname{cols}^{20}$ compararon los niveles de homocisteinemia a las 16 semanas de gestación en 52 mujeres que desarrollaron posteriormente preeclampsia y 56 que se mantuvieron normotensas. Encontraron niveles mayores mayores de 5,5 micromol/L (por encima del percentil 95 del valor control normal) en $29 \%$ de las preeclámpticas comparado con $13 \%$ de las que se mantuvieron normotensas. Además, se encontró que niveles elevados de homocisteinemia en el segundo trimestre fueron factor de riesgo para el desarrollo de preeclampsia: RD 3,2 (CI 95\% 1,1 - 9,2).

Powers y col ${ }^{16}$ compararon 21 pacientes preeclámpticas con 33 gestantes normales y encontraron niveles significativamente más altos de hmocisteinemia entre las enfermas $(\mathrm{p}<0,04)$. Además los niveles de homocisteinemia se relacionaron con los de fibronectina celular, un marcador de lesión endotelial, lo cual sugirió que niveles altos de homocisteinemia se asocian con la lesión endotelial que se presenta en la preeclampsia ( $\mathrm{p}<0,001)$.

Leeda y cols ${ }^{21}$ estudiaron 207 pacientes con preeclampsia o RCIU a las cuales realizaron test de carga de metionina y encontraron que $37 \quad(17,8 \%)$ tenían hiperhomocisteinemia; todas ellas fueron tratadas con ácido fólico (5 mg/día, vía oral) y vitamina $B_{6}(100 \mathrm{mg} /$ día vía oral). 27 que fueron reevaluadas 10 semanas después, tuvieron nuevo test de carga de metionina dentro de límites normales. Catorce se embarazaron nuevamente $\mathrm{y}$ recibieron ácido fólico, vitamina $\mathrm{B}_{6} \mathrm{y}$ ácido acetil salicílico (ASA) después de las 12 semanas. Estas pacientes tuvieron hijos con mayor peso al nacer comparados con los de su embarazo previo. Se sugirió que el suplemento con estos medicamentos puede ser útil en el manejo de las pacientes con hiperhomocisteinemia.

La medición de homocisteína y la definición de hiperhomocisteinemia no son homogéneas en los centros donde se realizaron las investigaciones mencionadas.

Los resultados de los ensayos clínicos indican que un porcentaje importante (25$33 \%$ ) de mujeres que experimentan abortos espontáneos y abrupcio de placenta presentan hiperhomocisteinemia basal $\mathrm{o}$ después del test de carga de metionina. La hiperhomocisteinemia se relaciona con una tasa elevada de muerte perinatal. En las gestantes afectas de homocistinuria clásica no tratada, generalmente con $\mathrm{HC} \geq$ a 50 micromol/L se presentan pérdidas fetales en el $50 \%$ de los casos, las demás presentan riesgo elevado de preeclampsia ${ }^{11}$.

\section{RECOMENDACIONES}

Acorde con nuestros resultados, es pertinente considerar 13,1 micromol/L como punto de corte para la definición de hiperhomocisteinemia en la gestante. La prueba puede realizarse cuantificando los niveles séricos posprandiales de homocisteina mediante el método inmunoenzimático descrito.

Es necesario realizar estudios controlados que busquen asociación causal entre hiperhomocisteinemia y preeclampsia. Adecuadamente aleatorizados, pueden seleccionarse casos y controles para definir el papel de la suplementación con Vitamina $\mathrm{B}_{6} \mathrm{y} / \mathrm{o}$ ácido fólico en la normalización de la hiperhomocisteinemia, en la prevención de la preeclampsia y sus complicaciones.

\section{AGRADECIMIENTOS}

Al Laboratorio Clínico Hematológico de Medellín, Colombia, por su generoso aporte para la culminación del proyecto.

\section{BIBLIOGRAFÍA}

1. E.U. Population Reference Bureau (PRB). Resumen de las conclusiones del Consejo Nacional de Investigación de la Academia Nacional de Ciencias de los E.U, "Cómo mejorar la salud reproductiva de los países en desarrollo". Washington; 1997.

2. Presidencia de la República, Departamento Nacional de Planeación, Ministerio de Salud y otros. "Mortalidad Materna en Colombia". Santafé de Bogotá; 1996.

3. Ness R, Roberts JM. Heterogeneous causes constituting the single syndrome of preeclampsia: A hypothesis and its implications. Am J Obstet Gynecol 1996;175:1365-70.

4. Stone J. Risk factors for severe preeclampsia. Obstet Gynecol;1994;83:357-61. 
5. Sibai B. Aspectos inmunitarios de la preeclampsia. Clín Obstét Ginecol 1991:27-33.

6. Lockwood Ch, Rand J. The immunobiology and obstetrical consequences of antiphospholipid antibodies. Obstet Gynecol Surv 1994;49:432-40.

7. Molina J. Anticuerpos Antifosfolípidos. IATREIA 1992;5:160-1

8. Dekker G, de Vries J, Doelitzsch P, et al. Underlying disorders associated with severe early-onset preeclampsia. Am J Obstet Gynecol 1995;173:1042-8.

9. Kupferminc M, Eldor A, Steinman N, et al. Increased frequency of genetic thrombophilia in women with complications of pregnancy. $\mathrm{N}$ Engl J Med 1999;340: 9-13.

10. Blummenfield Z, Brenner B. Thrombophilia-associated pregnancy wastage. Fertil Steril 1999;72:765-74.

11. Córdoba A, Blanco F, González F. Hiperhomocisteinemia, un nuevo marcador de riesgo vascular: territorios vasculares afectados; papel en la patogénesis de la arterioesclerosis y la trombosis y tratamiento. Med Clin (Barc) 1997;109:715-25.

12. Pampus M, Wolf $H$, Buller $H$. Underlying disorders associated with severe preeclampsia and Hellp syndrome. Am J Obstet Gynecol 1997;176:S26

13. Rajkovic A, Mahomed K, Malinov R. Elevated homocysteine levels with preeclampsia. Obstet Gynecol 1997;90:168-71. 14. Rajkovic A, Mahomed K, Malinov R, et al. Plasma homocysteine concentrations in eclamptic and preeclamptic African women postpartum. Obstet Gynecol 1999;94:355-60.

15. Laivuori H, Kaaja R, Turpeinem U, et al. Plasma homocystein levels elevated and inversely related to insulin sensitivity in preeclampsia. Obstet Gynecol 1999;93:489-93.

16. Powers R, Evans R, Majors A, et al. Plasma homocystein concentration is increased in preeclampsia and is associated with evidence of endothelial activation. Am J Obstet Gynecol 1998;179:1605-11.

17. Raijmakers M, Zusterzeel P, Steegers E, et al. Plasma Thiol status in preeclampsia. Obstet Gynecol 2000;95:180-4.

18. Ray JG, Laskin CA. Folic acid and homocystein metabolic effects and risk of placental abruption, preeclampsia and spontaneous pregnancy loss. A systematic review. Placenta; 1999;20:519-29.

19. Wang J, Trudenger BJ, Duarte N. Elevated circulating homocystein levels in placental vascular disease and associated preeclampsia. Br J Obstet Gynecol; 2000;107:935-8.

20. Sorensen TK, Malino MR, Williams MA. Elevated second trimester serum homocystein levels and subsequents risk of preeclampsia. Gynecol Obstet Invest; 1999;48:98-103.

21. Leeda M, Riyas I, de Vries JIP, et al. Effects of folic acid and vitamin $\mathrm{B}_{6}$ supplementation on women with hyperhomocysteinemia and history of preeclampsia or fetal grow restriction. Am J Obstet Gynecol 1997;179:135-9. 